\title{
Dynamic localization in optical and Zeeman lattices in the presence of spin-orbit coupling
}

\author{
Yaroslav V. Kartashov ${ }^{1,2}$, Vladimir V. Konotop ${ }^{3}$, Dmitry A. Zezyulin ${ }^{3}$, and Lluis Torner $^{1,4}$ \\ 1 ICFO-Institut de Ciencies Fotoniques, The Barcelona Institute \\ of Science and Technology, 08860 Castelldefels (Barcelona), Spain \\ ${ }^{2}$ Institute of Spectroscopy, Russian Academy of Sciences, Troitsk, Moscow Region, 142190, Russia \\ ${ }^{3}$ Centro de Física Teórica e Computacional Faculdade de Ciências and Departamento de Física, \\ Faculdade de Ciências, Universidade de Lisboa, Campo Grande, Ed. C8, Lisboa 1749-016, Portugal \\ 4 Universitat Politecnica de Catalunya, 08034 Barcelona, Spain
}

(Dated: November 18, 2016)

\begin{abstract}
The dynamic localization of a two-level atom in a periodic potential under the action of spinorbit coupling and a weak harmonically-varying linear force is studied. We consider optical and Zeeman potentials that are either in-phase or out-of-phase in two spinor components, respectively. The expectation value for the position of the atom after one oscillation period of the linear force is recovered in authentic resonances or in pseudo-resonances. The frequencies of the linear force corresponding to authentic resonances are determined by the band structure of the periodic potential and are affected by the spin-orbit coupling. The width/dispersion of the wavepacket in authentic resonances is usually minimal. The frequencies corresponding to pseudo-resonances do not depend on the type of potential and on the strength of the spin-orbit coupling, while the evolution of excitations at the corresponding frequencies is usually accompanied by significant dispersion. Pseudo-resonances are determined by the initial phase of the linear force and by the quasi-momentum of the wavepacket. Due to the spinor nature of the system, the motion of the atom is accompanied by periodic, but not harmonic, spin oscillations. Under the action of spin-orbit coupling the oscillations of the wavepacket can be nearly completely suppressed in optical lattices. Dynamic localization in Zeeman lattices is characterized by doubling of the resonant oscillation periods due to band crossing at the boundary of the Brillouin zone. We also show that higher harmonics in the Fourier expansion of the energy band lead to effective dispersion, which can be strong enough to prevent dynamic localization of the Bloch wavepacket.
\end{abstract}

PACS numbers: 37.10.Jk, 32.60.+i, 03.75.-b

\section{INTRODUCTION}

A linear force applied to a quantum particle in a periodic potential does not lead to unidirectional motion, but results in particle oscillations. This is the celebrated phenomenon of Bloch oscillations known since the early days of quantum mechanics [1]. Bloch oscillations have been experimentally observed in multiple solid-state settings [2], in atomic systems 3, 4], and in optics 5] (for reviews of the current state of the art, see [6]). An oscillatory motion, or spatial localization, of a particle in a periodic potential can also be caused by a linear force whose amplitude oscillates with a properly selected frequency. This phenomenon, known as dynamic localization, was discovered in Ref. 7] within the framework of the tightbinding approximation widely used in physics. It was later studied in systems of cold atoms held in optical lattices (OLs) 8], where it was used for the coherent control of atoms and for realization of the superfluid - Mott insulator phase transition [9]. Dynamic localization occurs also in optical systems 10], where a periodic waveguide bending may emulate a time-periodic linear force acting on a quantum particle. The effect can be used, e.g., to cause rectification of beam propagation trajectories [11].

Dynamic localization occurs also in nonlinear systems. In the theory of integrable systems, the dynamics of solitons in special nonlinear lattices (including those gov- erned by the Ablowitz-Ladik model [12]) subject to timedependent linear forces was addressed in Ref. 13] even before the phenomenon of dynamic localization caused by an oscillating force. Actually, the linear limit of the above mentioned model is the tight-binding approximation used in Ref. 7] and in many subsequent studies of dynamic localization. The oscillatory motion of solitons affected by a time-periodic linear force as a manifestation of their dynamic localization was discussed in [14]. The interplay between dynamic localization and nonlinearity has been also studied in the meanfield theory of Bose-Einstein condensates loaded in optical lattices, both theoretically [15] and experimentally [16].

It should be properly appreciated that when compared with Bloch oscillations, dynamic localization offers more opportunities for controlling the particle dynamics (or, in optics, the light propagation). Indeed, it allows changing the direction of motion and average velocity of the particles simply by varying the frequency of the linear force. In contrast, a constant force leads only to oscillatory dynamics and the amplitude of the force affects only the amplitude of the Bloch oscillations.

All studies mentioned above deal with dynamic localization of electrons, atoms, or optical wavepackets described by a one-component wavefunction or, alternatively, by a single quantum number (quasi-momentum). In this paper we study dynamic localization of two- 
level atoms characterized by a spinor wavefunction. The quantum numbers describing such atom are the quasimomentum and (pseudo-)spin.

The systems of effective two-level atoms are attracting increasing attention due to the proven experimental possibility of creation of synthetic electric [17] and magnetic [18, 19] fields in them, which opens new avenues for the investigation of the physics of gauge potentials [20]. One of the most significant advances in this direction is the creation of spin-orbit (SO) coupled Bose-Einstein condensates (BECs) [21] [see 22] for a review], where periodic potentials can be induced not only in the form of OLs [3], equal for both spinor components, but also in the form of Zeeman lattices (ZLs) [23], which are out-ofphase for two spinor components.

Studies of Bloch oscillations of SO coupled atoms and BECs loaded in OLs 24 27] and in ZLs [26, 27], have already been reported. Here we address the impact of a time-periodic modulation of the amplitude of the linear force on the dynamics of two-level SO-coupled atoms. The problem and main analytical results are introduced in Sec. III In Sections [II and IV we describe the phenomenon of dynamic localization for an atom loaded in an OL and in a ZL, respectively. The results are summarized in the Conclusions section.

\section{MODEL}

We consider a two-level atom characterized by a spinor $|\Psi\rangle=\Psi=\left(\Psi_{1}, \Psi_{2}\right)^{T}$ (hereafter the superscript " $T$ " stands for transposition). Throughout the paper we deal with periodic potentials described by the matrix

$$
h_{\epsilon}(x)=\epsilon V(x) I+(1-\epsilon) \Omega_{3}(x) \sigma_{3} .
$$

Here $\sigma_{j}(j=1,2,3)$ are the standard Pauli matrices, $I$ is the $2 \times 2$ unit matrix, $\epsilon=1$ corresponds to an OL $h_{1}=$ $V(x) I$, while $\epsilon=0$ corresponds to a ZL $h_{0}(x)=\Omega_{3}(x) \sigma_{3}$. The dimensionless spatial coordinate $x$ is measured in the units $L / \pi$ where $L$ is the lattice period in the physical units. Thus, both lattices, i.e. both functions $V(x)$ and $\Omega_{3}(x)$, are $\pi$-periodic, $h_{\epsilon}(x)=h_{\epsilon}(x+\pi)$. We also assume that the lattices are even with respect to the origin, i.e, $h_{\epsilon}(x)=h_{\epsilon}(-x)$, and odd with respect to the quarterperiod, i.e., $h_{\epsilon}(\pi / 4+x)=-h_{\epsilon}(\pi / 4-x)$. For instance, one can consider $\left\{V, \Omega_{3}\right\} \propto \cos (2 x)$. In the units, where $\hbar=1$ and $m=1 / 2$, the evolution of the spinor $|\Psi\rangle$ is described by the Schrödinger-Pauli equation:

$$
i \frac{\partial|\Psi\rangle}{\partial t}=H|\Psi\rangle+\beta(t) x|\Psi\rangle, \quad H \equiv H_{0}+h_{\epsilon}(x),
$$

where

$$
H_{0}=p^{2}+\gamma \sigma_{3} p+\Omega_{1} \sigma_{1},
$$

$p=-i \partial_{x}$ is the operator of linear momentum, $\gamma$ is the strength of the SO coupling, and $2 \Omega_{1}$ is the Rabi frequency (further we set $\Omega_{1}=0.5$ ). The term $\beta(t) x$ takes into account the linear force oscillating with the frequency $\omega$ and having zero mean value on one oscillation period.

We are interested in the evolution of the wavepacket center which can be characterized by its coordinate $x_{c}$ defined as the expectation value

$$
x_{c}=\langle\Psi|x| \Psi\rangle=\int_{-\infty}^{\infty} \Psi^{\dagger} x \boldsymbol{\Psi} d x .
$$

Below we use the complete set of Bloch states $|k, \alpha\rangle$ of the Hamiltonian $H: H|k, \alpha\rangle=\mu_{\alpha}(k)|k, \alpha\rangle$, where $\mu_{\alpha}(k)$ is the energy of the state, the Bloch quasi-momentum $k$ belongs to the reduced Brillouin zone (BZ): $k \in(-1,1]$, and $\alpha=1,2, \ldots$ is the band number ( $\alpha=1$ being the number of the lowest band). Following Houston's approach [28], we introduce adiabatically-varying Bloch states $|\kappa(t), \alpha\rangle$ where

$$
\kappa(t)=k+B(t), \quad B(t)=\int_{0}^{t} \beta\left(t^{\prime}\right) d t^{\prime},
$$

and $k$ is the initial value of the quasi-momentum (or central quasi-momentum in the case of localized Bloch wavepackets). The spinor $|\Psi\rangle$ can be expanded in terms of the adiabatically-varying states as:

$$
|\Psi\rangle=\sum_{\alpha=1}^{\infty} \int_{-1}^{1} d k \chi_{\alpha}(k, t)|\kappa(t), \alpha\rangle .
$$

and, for the sake of convenience, the spectral coefficients will be represented as

$$
\chi_{\alpha}(k, t)=\chi_{\alpha}^{(0)}(k, t) e^{-i \int_{0}^{t} \mu_{\alpha}[\kappa(\tau)] d \tau}
$$

with the functions $\chi_{\alpha}^{(0)}(k, t)$ to be determined latter. The normalization condition (in the direct and Fourier spaces)

$$
\langle\Psi \mid \Psi\rangle=\sum_{\alpha=1}^{\infty} \int_{-1}^{1}\left|\chi_{\alpha}(k, t)\right|^{2} d k=1
$$

is also imposed.

We assume that the following conditions hold:

Condition 1: Bloch states of only one band, say of the band $\alpha_{0}$, are initially excited, i.e. $\chi_{\alpha}(k, 0)=0$ for $\alpha \neq \alpha_{0}$.

Condition 2: A wavepacket $|\Psi\rangle$ is a Bloch wave with a smooth and broad envelope (compared to the lattice period) and its spectrum centered at a quasi-momentum $k_{0}$ in the reduced $\mathrm{BZ}$ is much narrower than the $\mathrm{BZ}$ zone, so that the approximation

$$
\int_{-1}^{1} d k\left|\chi_{\alpha_{0}}(k, t)\right|^{2} \frac{\partial \mu_{\alpha_{0}}(k)}{\partial k} \approx \frac{\partial \mu_{\alpha}\left(k_{0}\right)}{\partial k_{0}}
$$

is valid [here we take into account the normalization (8)] .

Condition 3: The linear force is weak, i.e., $|\beta(t)| \ll 1$, so that inter-band tunneling is negligible.

Under these conditions one can show that the the dynamics of the wavepacket center is governed by the equation (see [27] for the derivation):

$$
\frac{d x_{c}}{d t}=\left.\frac{\partial \mu_{\alpha_{0}}(k)}{\partial k}\right|_{k=k_{0}+B(t)},
$$


where $k_{0}$ is the initial central quasi-momentum of the wavepacket.

Taking into account that due to Floquet theorem the energy is a periodic function, with period $2, \mu_{\alpha_{0}}(k)$ can be expanded in a Fourier series. Generally, if the excited band $\alpha_{0}$ is narrow enough, one can restrict the consideration to only one harmonic associated with nonzero frequency - this is a standard assumption in the tightbinding approximation. However, in our system with SO coupling this is usually not the case (see Fig. 1 with representative band structures), and one has to take into account several Fourier harmonics in the expansion

$$
\mu_{\alpha}(k)=\sum_{m=0}^{\infty} \mu_{\alpha}^{(m)} \cos (m \pi k) .
$$

(In order to simplify analytical expressions, in (11) as well as in the subsequent formulas we omit subscript 0 at $\alpha$ and write $\alpha$ instead of $\alpha_{0}$ bearing in mind that only one zone is being considered.) This yields the final form of the equation describing the dynamics of the center of the wavepacket:

$$
\frac{d x_{c}}{d t}=-\pi \sum_{m=1}^{\infty} m \mu_{\alpha}^{(m)} \sin \left\{m \pi\left[k_{0}+B(t)\right]\right\} .
$$

Now we consider a periodic force $B(t)=B(t+T)$, with the period $T$ and frequency $\omega=2 \pi / T$. Dynamic localization is characterized by the vanishing average velocity of the wavepacket, where averaging is performed over the period $T$, as well as by restoration of the wavepacket shape after each period, i.e., by suppression of dispersion. Thus the resonant frequency $\omega$ at which the dynamic localization occurs [i.e. at which $x_{c}(0)=x_{c}(T)$ ] is determined from the condition

$$
\sum_{m=1}^{\infty} m \mu_{\alpha}^{(m)} \int_{0}^{T} \sin \left\{m \pi\left[k_{0}+B(t)\right]\right\} d t=0
$$

First, we consider a general harmonic force of the form $\beta(t)=\beta_{0} \sin (\omega t+\varphi)$ where $\beta_{0}$ and $\varphi$ are the amplitude and the initial phase of the force. Now the integrals in (13) are computed explicitly resulting in the condition

$$
\begin{aligned}
& \mathcal{J}\left(\omega, k_{0}\right) \equiv \sum_{m=1}^{\infty} m \mu_{\alpha}^{(m)} \sin [\left.\pi m\left(k_{0}+\frac{\beta_{0}}{\omega} \cos \varphi\right)\right] \\
& \times J_{0}\left(\frac{m \pi \beta_{0}}{\omega}\right)=0,
\end{aligned}
$$

where $J_{0}(\cdot)$ is the zero-order Bessel function of the first kind. Equation (14) will be used below for the comparison of analytical predictions with the results of direct numerical simulations.

An immediate outcome of (14) follows from the fact that $\sin (m \nu)=\sin \nu U_{m-1}(\cos \nu)$, where $U_{m}(\cdot)$ is the Chebyshev polynomial of the second kind: independently of the type of the lattice, i.e. independently of $\epsilon$ in Eq. (1), the sum in Eq. (14) is proportional to

$$
\sin \left[\pi\left(k_{0}+\frac{\beta_{0}}{\omega} \cos \varphi\right)\right] .
$$

Thus the function $\mathcal{J}\left(\omega, k_{0}\right)$ vanishes at the frequencies $\omega=\tilde{\omega}_{n}$ defined as

$$
\tilde{\omega}_{n}=\frac{\beta_{0} \cos \varphi}{n-k_{0}}
$$

where $n$ is an integer. These zeros, however do not represent authentic resonances corresponding to the dynamic localization, but reflect the adiabatic theorem [30] ensuring variation of the quasi-momentum caused by slowly varying weak external force. The zeros $\tilde{\omega}_{n}$ depend on the central momentum $k_{0}$ (as well as on the initial phase of the force $\varphi$ ) and thus at $\omega=\tilde{\omega}_{n}$ the dynamics is accompanied by the effective dispersion of the wavepacket. To separate the resonances at $\tilde{\omega}_{n}$ from the authentic resonances $\omega_{k}$ leading to the dynamic localization, the former ones will be termed pseudo-resonances. When only one harmonic $(m=1)$ is taken into account in the expansion of the band energy into Fourier series, authentic resonances can be found using zeros of the Bessel functions in Eq. (14). In this case $\omega_{k}$ are approximately given by $\omega_{k}=\pi \beta_{0} / \nu_{k}$, where $\nu_{1} \approx 2.405, \nu_{2} \approx 5.520$, etc., are zeros of $J_{0}(\nu)$. In the authentic resonances computed within this tight-binding approximation the dispersion usually vanishes too and the wavepacket undergoes periodic in time localization.

We concentrate on the case $\varphi=0$, i.e.,

$$
\beta(t)=\beta_{0} \sin (\omega t),
$$

and consider initial wavepackets with $k_{0}=0$ exciting modes in the center of the first BZ. Due to relative smoothness of the band structure (see Fig. 1 below), for accurate description of all resonances it is usually enough to take into account only a few harmonics in (14) (see Tables \and [1 below). Thus, the resonant frequencies at which dynamic localization occurs are found from the equation

$$
\begin{aligned}
\mathcal{J}_{M}(\omega) & \equiv \mu_{\alpha}^{(1)} J_{0}\left(\frac{\pi \beta_{0}}{\omega}\right) \\
+ & \sum_{m=2}^{M} m \mu_{\alpha}^{(m)} \frac{\sin \left(m \pi \beta_{0} / \omega\right)}{\sin \left(\pi \beta_{0} / \omega\right)} J_{0}\left(\frac{m \pi \beta_{0}}{\omega}\right) .
\end{aligned}
$$

The expressions (18) [or (14)] reveal a noticeable result: the more complex the dependence $\mu_{\alpha}(k)$, i.e. the larger the number of Fourier harmonics needed for its accurate approximation, the more pronounced is the effective dispersion. In order to estimate the variation of the width of the wavepacket, we assume that initially it has the width $d_{0}$, and hence its spectral width can be defined as $\delta k=2 \pi / d_{0}$. Since resonant frequencies depend on the quasi-momentum, one can estimate the difference 
between resonant periods of the linear force for excitations separated by $\delta k$ in the frequency domain as:

$$
\delta T \approx-\frac{2 \pi}{\omega^{2}}\left(\frac{d \omega}{d k}\right)_{k_{0}} \delta k=\frac{4 \pi^{2}}{\omega^{2} d_{0}} \frac{\partial \mathcal{J}\left(\omega, k_{0}\right) / \partial k_{0}}{\partial \mathcal{J}\left(\omega, k_{0}\right) / \partial \omega},
$$

where $\mathcal{J}(\omega, k)$ was defined in (14) and the derivative of $d \omega(k) / d k$ was computed using the implicit relation $\mathcal{J}(\omega, k)=0$. Respectively, the variation of the wavepacket width over one period of the linear force can be estimated as the absolute value of the product of the difference of the resonant periods $\delta T$ for two harmonics separated by $\delta k$ and the difference of their group velocities:

$$
\left(\frac{d \mu_{\alpha}(k)}{d k}\right)_{k_{0}}-\left(\frac{d \mu_{\alpha}(k)}{d k}\right)_{k_{0}+\delta k} .
$$

Since in our case $k_{0}=0$ and $d \mu_{\alpha}(k) /\left.d k\right|_{k=0}=0$ the required estimate reads

$$
\delta d \approx \delta T \cdot\left(\frac{d \mu_{\alpha}(k)}{d k}\right)_{\delta k} \approx \frac{(2 \pi)^{3} \mu_{\alpha}^{(1)}}{\omega^{2} d_{0}^{2}}\left(\frac{\mathcal{J}_{k}(\omega, k)}{\mathcal{J}_{\omega}(\omega, k)}\right)_{k=0}
$$

Here we took into account only the first harmonic in the expansion (11) of $\mu_{\alpha}(k)$ in the Fourier series.

In the tight-binding approximation, where only one Fourier harmonic is taken into account, i.e. $M=1$, one readily gets $(d \omega / d k)_{k=k_{0}}=0$, i.e. $\delta d=0$ as described in many previous studies.

\section{DYNAMIC LOCALIZATION IN OPTICAL LATTICES}

First we consider an atom in an OL where the Hamiltonian in Eq. (2) takes the form $H=H_{0}+h_{1}(x)=$ $H_{0}+V(x) I$. In all numerical simulations we consider $V(x) \equiv-4 \cos (2 x)$ and concentrate on an atom whose energy belongs to one of the two lowest bands, shown in Fig. 1(a) and Fig. 1(b) for several values of the SO coupling. In these panels one observes well-known effects 27, 29] such as collapse of the band width (red curves 2) and inversion of the curvature (seen from the comparison of the curves 1 and 3) upon increase of the strength of the SO coupling. It is this effect that drastically affects the phenomenon of dynamic localization in SO-coupled systems, since according to Eq. (10) the amplitude of oscillations of the wavepacket under the action of a periodic linear force is determined by the width of the excited band. The widths of the first two bands in the spectrum are shown in Fig. 2(a) as functions of the SOcoupling strength $\gamma$. Notice that both first and second bands nearly collapse for a pair of $\gamma$ values, but these pairs are different for the first and second bands. For large values of $\gamma$ the width of the band oscillates nearly periodically.

Now we turn to the numerical simulations of the dynamic localization. To this end, we focus on the particular amplitude of the linear force $\beta_{0}=1 /(17 \pi) \approx 0.019$.
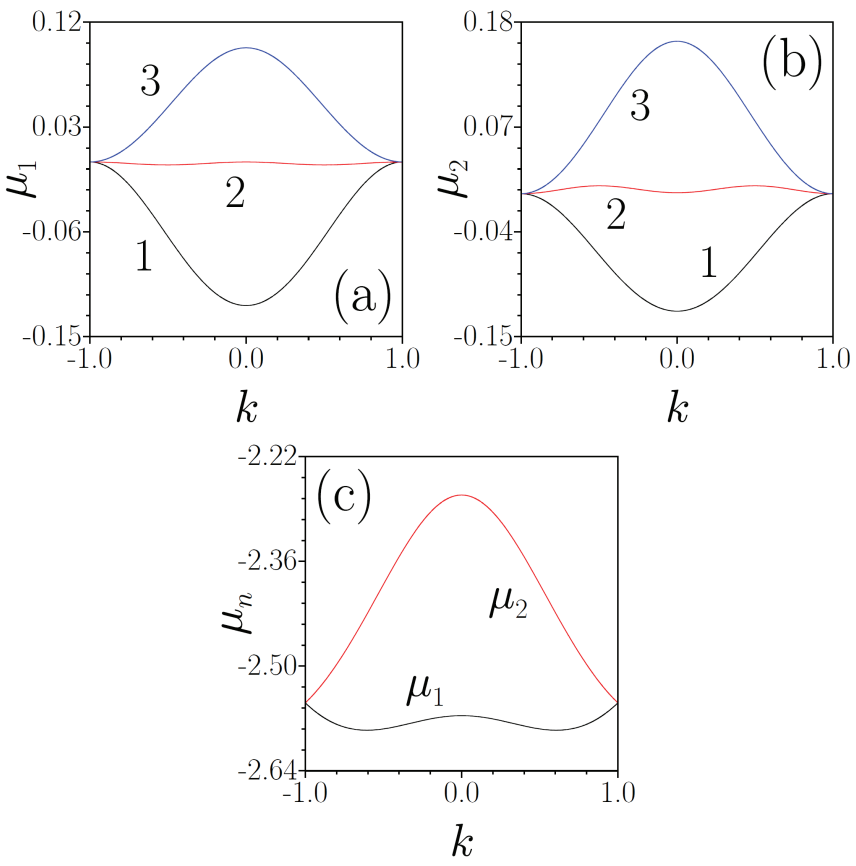

FIG. 1. (Color online) Transformation of the first (a) and second (b) bands in the spectrum for an OL with growing $\gamma$. In (a) $\gamma=0$ for curve $1, \gamma=1.17$ for curve 2 , and $\gamma=2.15$ for curve 3. In (b) $\gamma=0$ for curve $1, \gamma=0.82$ for curve 2, and $\gamma=1.84$ for curve 3 . The vertical shift of the bands with increasing $\gamma$ were eliminated by subtracting $\mu_{n}(k=1)$ from the $\mu_{n}(k)$ dependence. (c) First two bands in the spectrum for a $\mathrm{ZL}$ at $\gamma=2$.

In Fig. 3 we illustrate the main result obtained for an OL in the form of resonant curves showing dependence of the shift of the wavepacket center $x_{c}$ and its integral width $d$ on the frequency of linear force calculated after three periods of the force oscillations, $t=3 T=6 \pi / \omega$. Here and in all simulations of evolution dynamics we use the initial wavepacket in the form of modulated Bloch wave from the center of the BZ, i.e.,

$$
|\Psi(t=0)\rangle=e^{-\left(2 x / d_{0}\right)^{2}}|0, \alpha\rangle,
$$

where $\alpha=1$ in panels (a),(b) and $\alpha=2$ in panel (c). The instantaneous width $d(t)$ of the wavepacket was computed using the inverse form-factor, i.e. as

$$
d(t)=\left[\int_{-\infty}^{\infty}\left(\left|\psi_{1}\right|^{4}+\left|\psi_{2}\right|^{4}\right) d x\right]^{-1},
$$

i.e. initially $d(0)=d_{0}$.

With red and black dots in Fig. 3(a) we show resonances and pseudo-resonances that for the first band can be clearly identified by comparing panels (a) and (b): while in the authentic resonances (red dots) we observe no displacement of the center of the wavepacket and complete restoration of its input width after three periods, in pseudo-resonances (black dots) we still do not observe displacement, but dispersion can be rather 

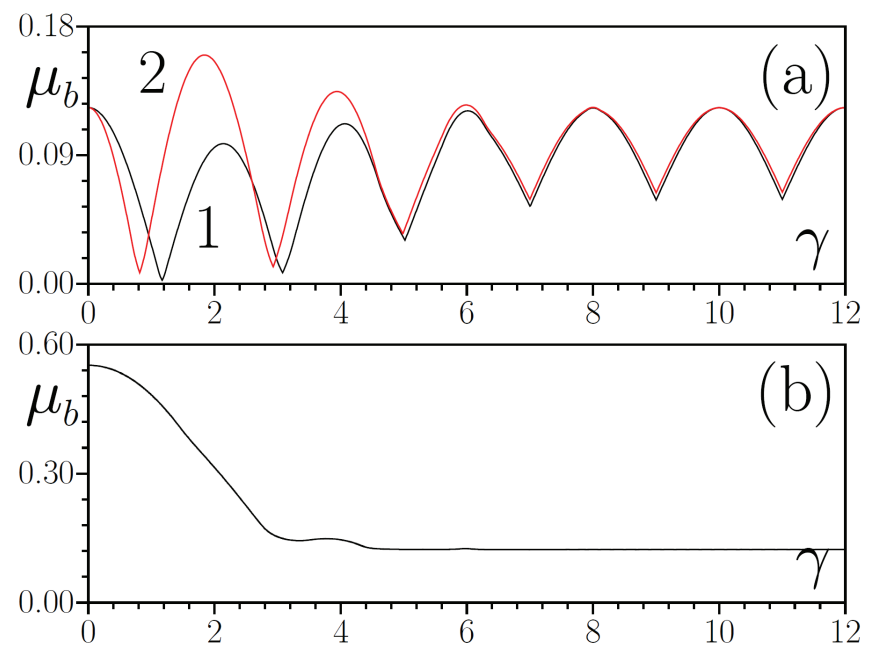

FIG. 2. (Color online) (a) Width of the first (curve 1) and second (curve 2) bands in an OL vs $\gamma$. (b) Width of the combined band in a ZL vs $\gamma$.

strong. Resonances and pseudo-resonances alternate: between two resonances one always encounter one pseudoresonance. Importantly, due to the collapse of the first band at $\gamma \approx 1.17$ and of the second band at $\gamma \approx 0.82$ [red curves 2 in Fig. 3(a) and (b), respectively], the oscillations of excitations from the corresponding bands are strongly suppressed, as well as their dispersion. In this regime (i.e., at these specific values of $\gamma$ ) the wavepacket propagates without broadening and displacement for any frequency $\omega$ of the linear force, so that dynamic localization does not occur. Notice also that the direction of the wavepacket displacement changes when the SO-coupling strength $\gamma$ passes the critical value corresponding to the band collapse [compare lines 1 and 3 in Fig. 3(a)]. Therefore, in this system one can control the direction of the wavepacket average displacement by changing the SOcoupling strength. The structure of resonances is similar for the first- and second-band excitations [compare panels Fig. 3(a) and 3(c)].

The resonant and pseudo-resonant frequencies obtained numerically agree remarkably well with the predictions based on the expression (18) even when only two harmonics in the Fourier expansion (i.e., $M=2$ ) are used. This is illustrated in Table I for several values of the SO coupling. It is relevant to mention that in spite of the smallness of the second harmonic $\left(\left|\mu_{\alpha}^{(2)}\right| \ll\left|\mu_{\alpha}^{(1)}\right|\right)$, taking it into account does improve the accuracy of the calculation of the resonant frequency. For example, when the first two resonance frequencies $\omega_{\text {num }}=0.02472$ and $\omega_{\text {num }}=0.0107$ at $\gamma=0$ are calculated using only one harmonic in the Fourier expansion (tight-binding approximation) one obtains $\omega_{1 \text { harm }}=0.02445$ and $\omega_{1 \text { harm }}=$ 0.01065 , respectively.

Different evolution scenarios of a Bloch wavepacket from the first band are shown in Fig. 4 for different values of the frequencies of the linear force. Taking into account

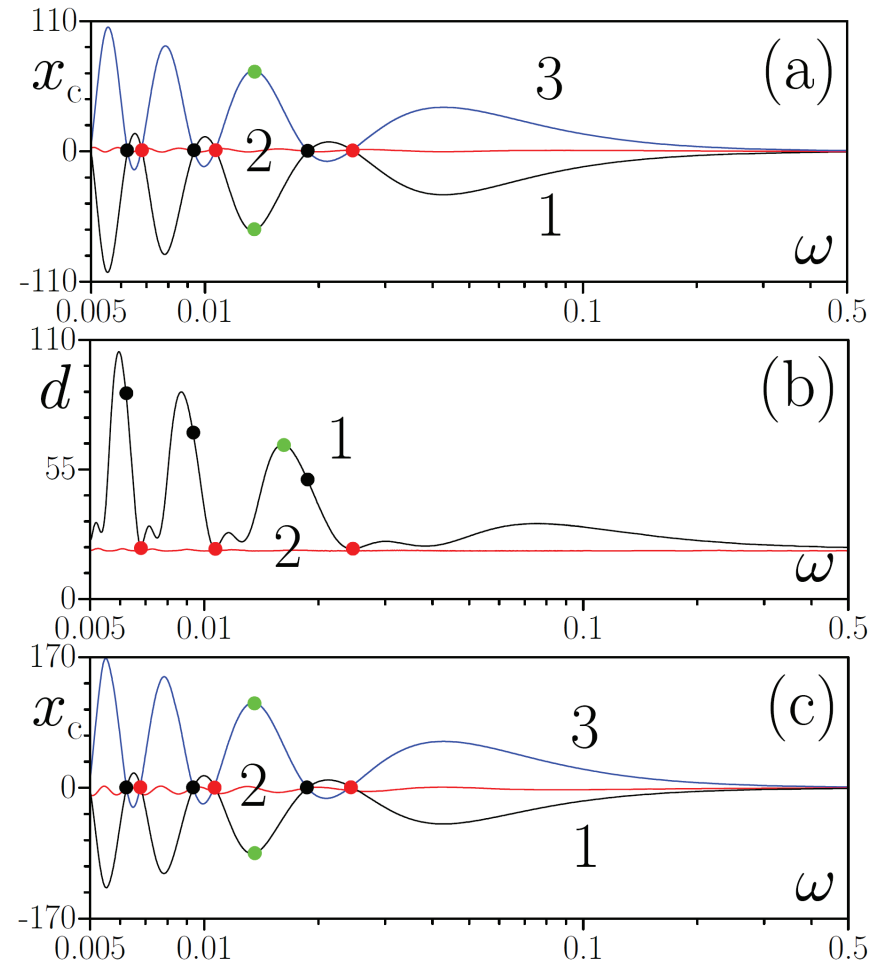

FIG. 3. (Color online) Resonant curves showing (a),(c) position of the integral center of the wavepacket and (b) its width at $t=6 \pi / \omega$ vs modulation frequency $\omega$ in the OL. Panels (a),(b) correspond to the first-band excitation at $\gamma=0.5$ (curve 1), $\gamma=1.17$ (curve 2), and $\gamma=2.15$ (curve 3 ). Panel (c) corresponds to the second-band excitation at $\gamma=0$ (curve 1), $\gamma=0.82$ (curve 2), and $\gamma=1.84$ (curve 3). Red dots show resonances where there is no drift and broadening of the wavepacket, while black dots show resonances where wavepacket broadens, but does not drift. Green dots mark frequencies at which largest displacement or broadening occurs between two first groups of resonances. In all cases $d(t=0)=7 \pi$. Notice the logarithmic scale in the horizontal axis.

the spinor nature of the wavefunction we show both the amplitude of the wavepacket (the upper row) and the expectation values for the spin components $S_{j}=\left\langle\Psi\left|\frac{1}{2} \sigma_{j}\right| \Psi\right\rangle$ (the lower row). Panels (a) and (b) show the evolution in the first authentic resonance and pseudo-resonance at $\gamma=0.5$ (see also Fig. 3(a), (b) and Table【). As mentioned above, in an authentic resonance the wavepacket experiences neither drift nor broadening, while in a pseudoresonance there is no drift, but one can observe considerable expansion. The drift of the wavepacket accompanied by slow dispersion at a non-resonant frequency is shown in panel (c), while complete suppression of oscillations and dispersion (even for a non-resonant frequency) in the case where band collapse occurs is illustrated in panel (d).

In all cases the motion of the Bloch wavepacket is accompanied by the spin-wave. A common property of all four panels in the lower row in Fig. 4 is that the spin 


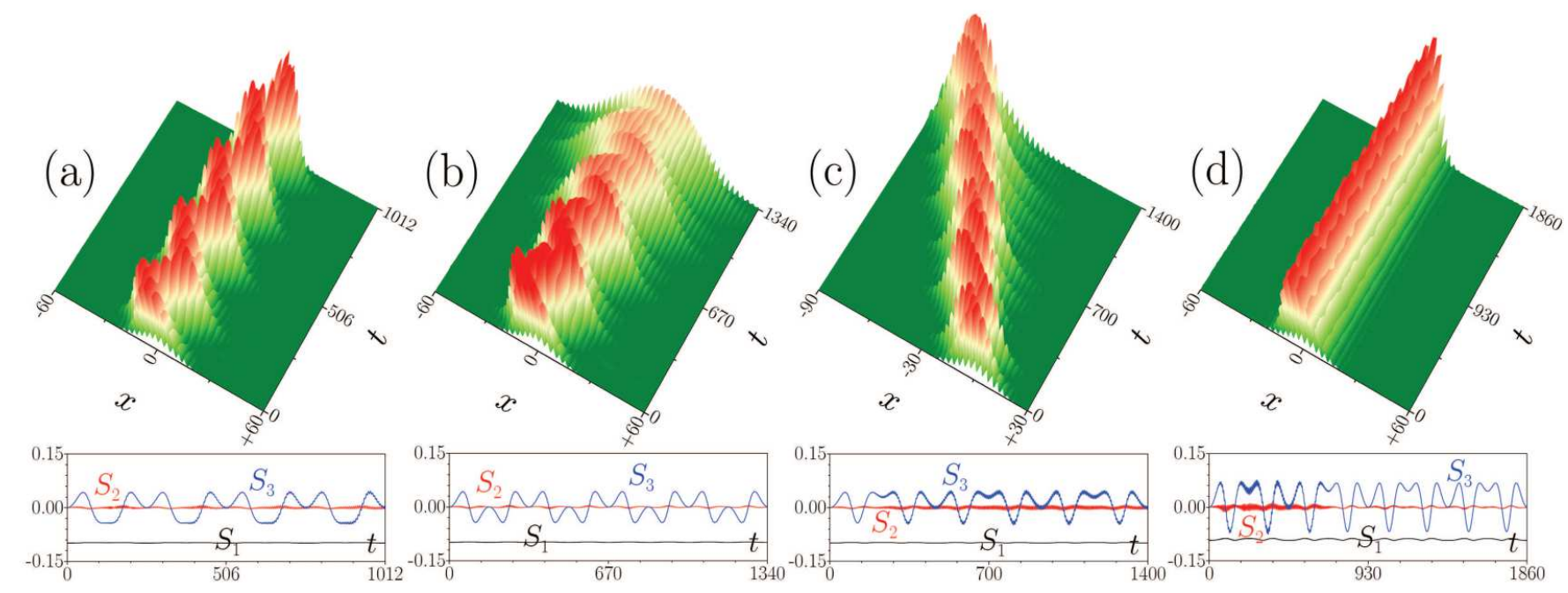

FIG. 4. (Color online) Evolution dynamics for the first-band excitations in an OL at (a) $\omega=0.0248, \gamma=0.5$, (b) $\omega=0.0187$, $\gamma=0.5$, (c) $\omega=0.0135, \gamma=0.5$, and (d) $\omega=0.0135, \gamma=1.17$. Only $\left|\psi_{1}\right|$ component is shown. The second row shows the corresponding evolution of the spin components. For illustrative purposes the magnitude of the $S_{1}$-component was divided by five. In all cases $d(t=0)=7 \pi$. In panels (a), (b), and (d) the evolution time corresponds to four complete oscillation periods, while in (c) it corresponds to three oscillation periods.

slightly oscillates in the $(x, z)$-plane with almost constant $x$-component and negligible $y$-component. This can be understood from the system of equations for the expectations of the spin components that admits the exact relation $d S_{3} / d t=2 \Omega_{1} S_{2}$, which for $S_{2}(t=0)=0$ leads to $S_{2} / S_{3} \sim 1 /\left(\Omega_{1} T\right)=\omega /\left(2 \pi \Omega_{1}\right)$. Thus the relation between the $y$ - and $z$-components is determined by the relation between the frequency of the linear force and the Rabi frequency. In the examples shown in Fig. 4, $\omega \sim 10^{-2}$ while $\Omega_{1}=0.5$. This leads to the estimate $S_{2} / S_{3} \sim 10^{-3}$ consistent with the negligible $y$-projection of the spin. Notice that the evolution of the $S_{3}$ component in "spin-up" $S_{3}>0$ and "spin-down" $S_{3}<0$ half-periods is different in all plots in Fig. (4) as a consequence of the broken parity symmetry in the $z$-direction due to the presence of the SO coupling.

In Fig. [5(a) and (b) we show the maximal displacement $x_{c}^{\max }$ (i.e. $\left|x_{c}^{\max }\right|$ is the amplitude of oscillations of the wavepacket center) and the maximal width of the wavepacket $d^{\max }$ achieved in the frequency interval between the first two authentic resonances, as the functions of the $\mathrm{SO}$ coupling. The values $x_{c}^{\max }$ and $d^{\max }$ were calculated numerically for excitations emerging from the first [Fig. [5(a)] and second [Fig. [5(b)] bands at $t=6 \pi / \omega$ (after three periods of the linear force). In Fig. 3 the maximal width of the wavepacket achieved are shown by green dots in the respective panels. Notice that the maximal amplitude and the maximal width are achieved at slightly different frequencies (visible in the logarithmic scale of Fig. (3)). The dependencies shown in Fig. 5(a) and 5(b) qualitatively reproduce band width from Fig. 2(a): the larger band width results in a larger amplitude of oscillations and larger dispersion of the wavepacket, while narrow band strongly suppresses both processes. We also observe that the closer the modulation frequency to one of the resonances, the smaller the smearing out of the probability density pattern due to dispersion. This implies that small deviations of modulation frequency from its resonant value do not result in a dramatic change of the dynamics.

To end this section, we recall that while the analytical results are general, all numerical simulations presented here were performed for specific values of the strength of the linear force and of the potential depth. However, we have verified that changing the values of such parameters does not introduce qualitative differences in the obtained results, as illustrated in Fig. 6. All resonant curves are qualitatively similar to the curves obtained for other parameters, as shown in Fig. 3. As it is expected from Eq. (14) [or from Eq. (18)] in Fig. 6 (a) one observes a shift of the resonances and pseudo-resonances towards larger frequencies as $\beta$ increases. Fig. 6 (b) shows that a change of the potential depth does not affect the location of the resonant frequencies, but it does significantly reduce the amplitude of the resonant curves [cf. Fig. 3 (a)]. This is in full agreement with the decrease of the band widths with increasing the potential depth. Therefore, one concludes that dynamic localization can be observed for a large range of lattice depths and linear forces, by tuning the frequency of modulation. 


\begin{tabular}{|c|c|c|c|c|}
\hline$\gamma$ & band & Fourier coefficients & $\omega_{2 h a r m}$ & $\omega_{\text {num }}$ \\
\hline & & \multirow{5}{*}{$\begin{array}{l}\mu_{1}^{(1)} \approx-0.0616 \\
\mu_{1}^{(2)} \approx-0.00127\end{array}$} & 0.02476 & 0.02472 \\
\hline & \multirow{4}{*}{1} & & 0.01872 & 0.01873 \\
\hline & & & 0.01071 & 0.01070 \\
\hline & & & 0.00936 & 0.00936 \\
\hline 0 & & & 0.00682 & 0.00682 \\
\hline & \multirow{5}{*}{2} & \multirow{5}{*}{$\begin{array}{l}\mu_{2}^{(1)} \approx-0.06160 \\
\mu_{2}^{(2)} \approx 0.00127\end{array}$} & 0.02476 & 0.02472 \\
\hline & & & 0.01872 & 0.01873 \\
\hline & & & 0.01071 & 0.01070 \\
\hline & & & 0.00936 & 0.00936 \\
\hline & & & 1.00682 & 0.00682 \\
\hline \multirow{10}{*}{0.5} & \multirow{5}{*}{1} & \multirow{5}{*}{$\begin{array}{l}\mu_{1}^{(1)} \approx-0.04802 \\
\mu_{1}^{(2)} \approx 0.00134\end{array}$} & 0.02487 & 0.0248 \\
\hline & & & 0.01872 & 0.01870 \\
\hline & & & 0.01073 & 0.01072 \\
\hline & & & 0.00936 & 0.00936 \\
\hline & & & 0.00683 & 0.00682 \\
\hline & \multirow{5}{*}{2} & \multirow{5}{*}{$\begin{array}{l}\mu_{2}^{(1)} \approx-0.03544 \\
\mu_{2}^{(2)} \approx-0.00172\end{array}$} & 0.02387 & 0.02472 \\
\hline & & & 0.01872 & 0.01872 \\
\hline & & & 0.01054 & 0.01056 \\
\hline & & & 0.00936 & 0.00937 \\
\hline & & & 0.00675 & 0.00675 \\
\hline \multirow{9}{*}{2.5} & & \multirow{5}{*}{$\begin{array}{l}\mu_{1}^{(1)} \approx 0.04067 \\
\mu_{1}^{(2)} \approx 0.00183\end{array}$} & 0.02391 & 0.02390 \\
\hline & & & 0.01872 & 0.01870 \\
\hline & 1 & & 0.01055 & 0.01055 \\
\hline & & & 0.00936 & 0.00936 \\
\hline & & & 0.00675 & 0.00675 \\
\hline & \multirow{4}{*}{2} & \multirow{4}{*}{$\begin{array}{l}\mu_{2}^{(1)} \approx 0.04541 \\
\mu_{2}^{(2)} \approx-0.00182\end{array}$} & 0.02506 & 0.02501 \\
\hline & & & 0.01872 & 0.01872 \\
\hline & & & 0.01077 & 0.01076 \\
\hline & & & $\begin{array}{l}0.00950 \\
0.00684\end{array}$ & 0.00684 \\
\hline
\end{tabular}

TABLE I. Coefficients of the Fourier expansion of the $\mu_{\alpha}(k)$ dependence; resonance and pseudo-resonance (in shadowed cells) frequencies $\omega_{2 \text { harm }}$ computed using two Fourier harmonics in the expansion (the coefficient $\mu_{0}$ does not contribute to dynamics), and resonant and pseudo-resonant frequencies $\omega_{\text {num }}$ obtained from the direct numerical simulations (see Fig. 3).

\section{DYNAMIC LOCALIZATION IN ZEEMAN LATTICES}

Now we consider the phenomenon of dynamic localization in a ZL. While formally the formulas derived in Sec. II remain valid (they were derived without any assumptions about the type of lattice potential), their physical interpretation has to be revised. The motivation is twofold: the wavepacket is two-component (spinor) and the bands of the ZL cross each other at the edges of the BZ [see Fig. 1(c)]. It was shown in [27] that when the wavepacket moves adiabatically along the selected branch of the band and arrives at the opposite edge of the BZ the associated spinor flips. In other words, unlike in an $\mathrm{OL}$, in the case of ZLs, after crossing the BZ a spinor does not recover its initial state. Moreover it does not continue moving in the initial band, but passes to the next band with which the initial band crosses at $k= \pm 1$. Let us assume that the indexes of the crossing bands are $\alpha$ and $\alpha+1$ [in Fig. 1 these are bands 1 and 2, i.e., $\alpha=1$ ].

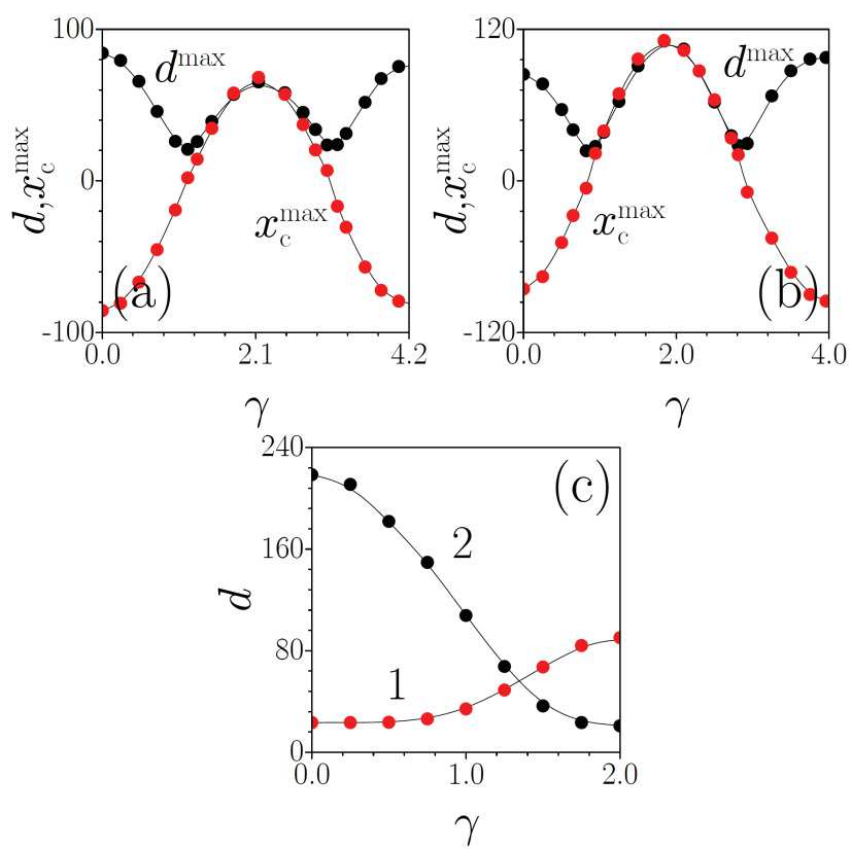

FIG. 5. (Color online) Maximal width of the wavepacket $d^{\text {max }}$ and its maximal displacement $x_{c}^{\max }$ between the two highest authentic resonances at $t=6 \pi / \omega$ vs $\gamma$ for the first-band (a) and second-band (b) excitations in the OL. In all cases $d(t=$ $0)=7 \pi$. (c) Width of the wavepacket at $t=6 \pi / \omega$ in the first (curve 1) and second (curve 2) resonances vs. $\gamma$ for the first-band excitation in the ZL. In all cases $d(t=0)=7 \pi$.

Suppose also that the initial wavepacket belongs to the $\alpha$-th band. Then, after the second crossing of the BZ but now in the band $\alpha+1$, the spin undergoes a new flip and the spinor returns to its original state. Hence, to compute the time of restoration of the wavepacket one has to consider the double crossing of the reduced BZ. Alternatively, and technically more convenient, one can consider the adiabatic motion in the extended BZ, which in our case is $k \in(-2,2]$. Then, instead of considering $\mu_{\alpha}(k)$ and $\mu_{\alpha+1}(k)$ in the reduced BZ, one considers

$$
\tilde{\mu}_{\alpha}= \begin{cases}\mu_{\alpha}(k), & k \in(-1,1] \\ \mu_{\alpha+1}(k), & k \in(-2,-1] \cup(1,2]\end{cases}
$$

and

$$
\tilde{\mu}_{\alpha+1}= \begin{cases}\mu_{\alpha+1}(k), & k \in(-1,1] \\ \mu_{\alpha}(k), & k \in(-2,-1] \cup(1,2] .\end{cases}
$$

Thus, $\tilde{\mu}_{\alpha}(k)=\mu_{\alpha+1}(k \pm 1)$. This means that the resonant and pseudo-resonant frequencies obtained from (18) [or (14)] must be divided by 2 to obtain the correct resonant frequencies in a ZL. Respectively, now one considers the Fourier expansion of $\tilde{\mu}_{\alpha}(k)$ over the extended BZ. Since profiles of the bands of ZLs in the extended BZ are more complex than those of OLs, one usually has to use three harmonics in the Fourier expansion for their accurate description. Keeping three harmonics in this expansion $(M=3)$, we computed the corresponding resonance frequencies for ZLs that are summarized in 

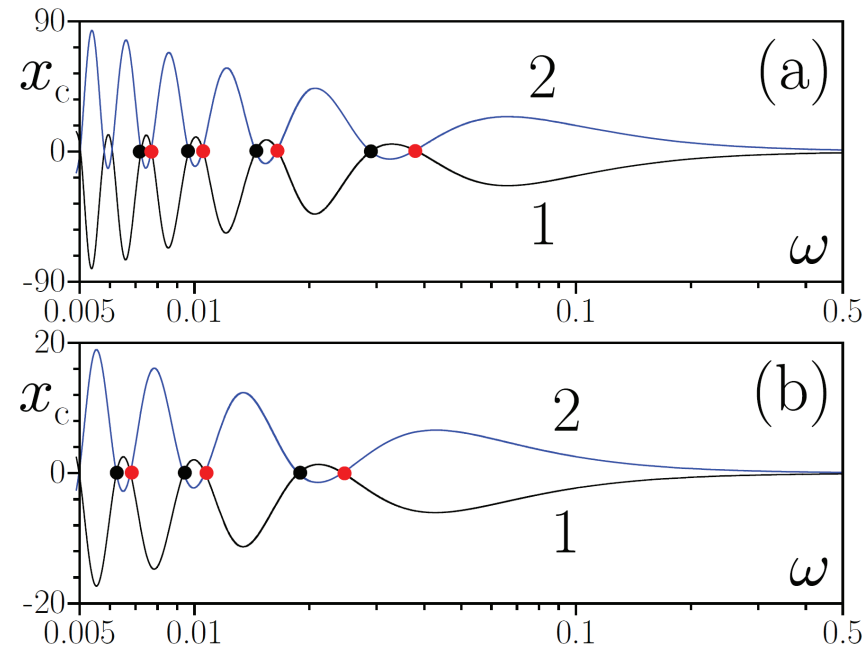

FIG. 6. (Color online) Resonant curves showing position of the integral center of broad wavepacket from the first band versus modulation frequency in the OL at $\gamma=0.5$ (curve 1) and $\gamma=2.15$ (curve 2). In (a) $\beta=1 / 11 \pi$ and lattice depth is 4 , while in (b) $\beta=1 / 17 \pi$ and lattice depth is 8 . Red dots - authentic resonances, black dots - pseudo-resonances.

Table II. Pseudo-resonant frequencies are now given by $\tilde{\omega} / 2$. It should be stressed here that even though the first and second bands cross each other [Fig. 1(c)], the coefficients in their expansion as a Fourier series for extended $\mathrm{BZ}$ are different and, therefore, the resonant frequencies obtained from (18) for the first and second bands are different too. This is readily apparent from Fig. 7 that illustrates completely different shapes and structure of resonances for the first and second band excitations.

The Table $\llbracket$ has a new column compared with Table【 which is the induced effective dispersion calculated using the estimate (20). Notice that in contrast to ZLs, in OLs all resonances correspond to strongly suppressed effective dispersion and to good localization of the wavepacket, and therefore effective dispersion is not included into Table I. Because of the relatively strong assumptions made upon calculation of the effective dispersion, the figures presented in Table II should be considered as approximations; they adequately characterize the dispersion after only one period of oscillations. Furthermore, since Eq. (20) is not applicable to pseudo-resonances, the respective cells are left empty.

We observe that the strength of the induced effective dispersion in ZLs may differ by an order of magnitude for different resonances. This allows us to classify resonances with suppressed dispersion $\delta d(T) \sim 1$ as highquality ones, while resonances with $\delta d(T) \gtrsim 10$ are lowquality resonances, where after only a couple of periods one observes delocalization of the wavepacket even though wavepacket displacement does not occur. In particular, for $\gamma=0$ high-quality resonances are found only for the first-band excitations, while all resonances for the second-band excitations are associated with strong dis-

\begin{tabular}{|c|c|c|c|c|c|}
\hline$\gamma$ & band & Fourier coefficients & $\omega_{3 h a r m}$ & $\omega_{\text {num }}$ & $\delta d(T)$ \\
\hline \multirow{8}{*}{0} & \multirow{4}{*}{1} & $\tilde{\mu}_{1}^{(1)} \approx-0.2831$ & 0.01169 & 0.0117 & 0.90000 \\
\hline & & $\begin{array}{l}\mu_{1} \sim-0.2001 \\
\tilde{\mu}^{(2)} \approx-00464\end{array}$ & 0.00936 & 0.00937 & - \\
\hline & & $\bar{\mu}_{1}^{2)} \approx-0.0464$ & 0.00522 & 0.00521 & 0.71442 \\
\hline & & $\mu_{1} \approx 0.0074$ & 0.00468 & 0.00469 & - \\
\hline & \multirow{4}{*}{2} & $\tilde{\mu}_{2}^{(1)} \approx 0.2831$ & 0.01364 & 0.01359 & 20.74133 \\
\hline & & $\tilde{\mu}_{2}(2) \approx 0.2001$ & 0.00936 & 0.00937 & - \\
\hline & & $\begin{array}{l}\mu_{2} \\
\tilde{u}_{(3)}\end{array}$ & 0.00557 & 0.00557 & 20.70126 \\
\hline & & & 0.00468 & 0.00469 & - \\
\hline \multirow{11}{*}{2} & \multirow{7}{*}{1} & \multirow{7}{*}{$\begin{array}{l}\tilde{\mu}_{1}^{(1)} \approx-0.14203 \\
\tilde{\mu}_{1}^{(2)} \approx 0.06511 \\
\tilde{\mu}_{1}^{(3)} \approx-0.00515\end{array}$} & 0.04689 & 0.04822 & 21.09505 \\
\hline & & & 0.01524 & 0.01525 & 28.42983 \\
\hline & & & 0.00969 & 0.00970 & 2.85704 \\
\hline & & & 0.00936 & 0.00936 & - \\
\hline & & & 0.00794 & 0.00797 & 23.55634 \\
\hline & & & 0.00581 & 0.00583 & 28.48675 \\
\hline & & & 0.00476 & 0.00473 & 2.86665 \\
\hline & \multirow{4}{*}{2} & $\tilde{\mu}_{2}^{(1)} \approx 0.14204$ & 0.01099 & 0.01095 & 3.48682 \\
\hline & & $\tilde{\mu}_{2}^{(2)} \approx 0.06511$ & 0.00936 & 0.00936 & - \\
\hline & & $\begin{array}{l}\mu_{2} \approx 0.00011 \\
\sim_{4}(3) \approx 00515\end{array}$ & 0.00506 & 0.00505 & 3.55544 \\
\hline & & $\tilde{\mu}_{2}^{\prime(v)} \approx$ & 0.00468 & 0.00468 & - \\
\hline
\end{tabular}

TABLE II. Coefficients of the Fourier expansion of the energy over the extended BZ for ZLs; resonance and pseudoresonance (in shadowed cells) frequencies $\omega_{3 h a r m}$ computed using three Fourier harmonics, the frequency $\omega_{\text {num }}$ obtained from the direct numerical simulations (see Fig. 7), as well as the estimate of the induced effective dispersion according to formula (20). Since the last formula generically is not valid for pseudo-resonances, the respective cells are left empty.

persion. In the case $\gamma=2$ the situation is somehow opposite: two highest resonances for the second-band excitation correspond to good localization, while for the first-band excitations only two out of six highest resonances are high-quality. Importantly, all predicted resonant frequencies as well as the orders of magnitude of the effective dispersion (directly manifested in the final width of the wavepacket) are confirmed numerically, as shown in Figs. 508 .

In order to show that the dispersion of the resonances strongly varies with an increasing SO-coupling strength, in Fig. 5(c) we plot the output width of the wavepacket calculated in two outermost right resonances from Fig. 7(a) (marked with red and black points). The wavepacket width in the authentic resonance (curve with red dots) gradually grows with $\gamma$, but decreases in pseudo-resonance (curve with black dots), so that at sufficiently large values of $\gamma$ pseudo-resonance appears to be "better" than the authentic one. Note also a significant difference in the resonant curves for the first- and secondband excitations in Fig. 7(a), which is explained by the different values of the Fourier harmonics in the series describing the shapes of the respective bands. Therefore, the induced effective dispersion can be potentially observed not only with two-level atoms in ZLs, but also in one-component systems, provided that they have band shapes that require for their accurate description more than one Fourier harmonic.

Finally, in Fig. 8 we show examples of the dynamics of Bloch wavepackets in ZLs subject to the periodic linear 


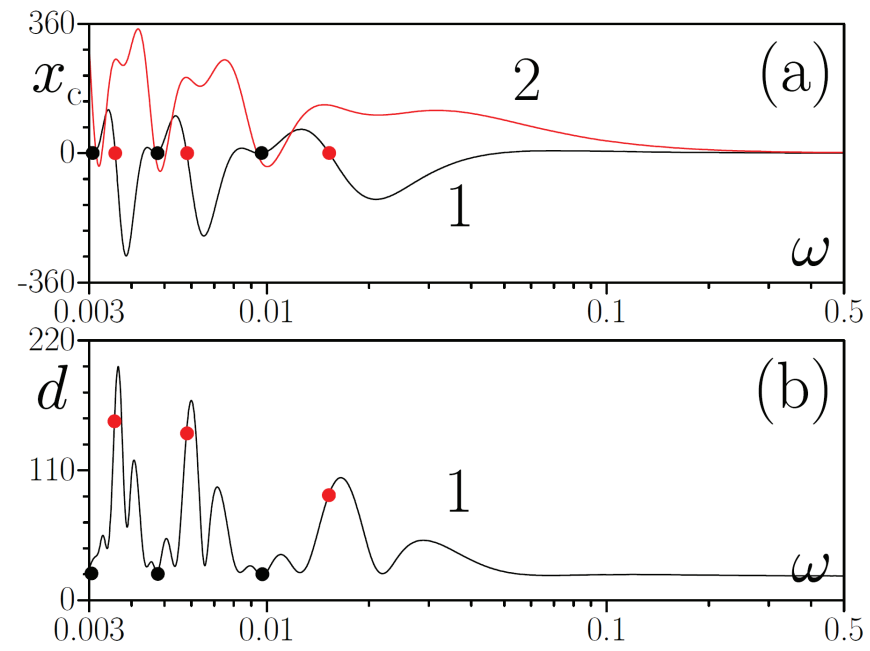

FIG. 7. (Color online) Resonant curves showing (a) position of the average center of the wavepacket and (b) its width at $t=6 \pi / \omega$ vs modulation frequency $\omega$ at $\gamma=2$ in the ZL. First-band (curve 1) and second-band (curve 2) excitations are shown in (a), while in (b) only first-band excitation is shown. Dots indicate resonances for the first-band excitation, which differ from resonances for second-band excitation. In all cases $d(t=0)=7 \pi$. The largest resonance 0.04822 (see Table II is not shown.

force. One observes more complex dynamics inside one period of oscillations, as compared to the case of OLs depicted in Fig. 4 which is related to the adiabatic motion of the wavepacket along the first and then along the second bands. The spin dynamics is different too. In the case of OLs the average spin exhibits a nearly constant and dominating $x$-component, in ZLs it rotates in the $(x, z)$ plane [the component $S_{2}$ is nearly negligible, as described in Sec. III for OLs and that remains valid for ZLs].

\section{CONCLUSIONS}

We addressed the phenomenon of dynamic localization of a spin-orbit-coupled two-level atom described by a spinor wavefunction, in optical and Zeeman lattices subjected to a periodically oscillating linear force. The analytical description of the phenomenon revealed a number of new features, some of which appear only for spinors while others were never discussed even in scalar one-component systems. The latter include the dependence of the dynamics on the phase of the external force, which may give rise to pseudo-resonances. Such pseudoresonance frequencies yield zero expectation values for the atom coordinate over one period. They disappear for specific phases of the oscillating force, do not depend on the strength of the spin-orbit coupling, and usually are associated with strong dispersion of the wavepacket.

We also found that for an arbitrary initial phase of the periodic force, a deviation of the band structure from the tight-binding limit (single harmonic in the Fourier spectrum) leads to induced effective dispersion. A practical consequence of this effect is the absence of complete localization, i.e., restoration of the initial shape of the wavepacket after one complete oscillation period of the linear force. It is worth mentioning that this phenomenon is not captured by the tight-binding approximation.

The effective dispersion allows distinguishing between high-quality and low-quality resonances: in the former, if the dispersion is strongly suppressed then one observes almost complete restoration of the initial shape of the wavepackets after several periods of oscillations, while in the latter resonances correspond to strong dispersion, even though the center of spinor wavepacket does not experience displacement. Since this phenomenon is determined by the deviation of the energy, i.e., $\mu_{\alpha}(k)$ dependence, from a cosine profile, it is strongly affected by the spin-orbit coupling that induces strong deformations of the band-gap structures.

The dynamics of the center of the wavepacket in the optical lattices does not depart significantly from teh behavior known for one-component wavefunctions, although now it is accompanied by rotation of the pseudo-spin. In the case of Zeeman lattices, the conclusion is drastically different. Indeed, bands in the Zeeman lattices cross each other and the spin of an wavefunction flips after one adiabatic passage of the Brillouin zone. Only after the second passage of the Brillouin zone, the spinor returns to its initial state. Therefore now the resonant frequencies are determined by the time necessary for the wavefunction to pass two Brillouin zones, rather than one. In all considered cases the numerically simulated dynamics agrees remarkably well with the analytical results.

\section{ACKNOWLEDGMENTS}

The work of VVK and DAZ was supported by the FCT (Portugal) grant UID/FIS/00618/2013. YVK and LT have been partially supported by the Severo Ochoa Excellence program and by Fundacio Cellex and Fundacio Mir-Puig Barcelona.
[1] F. Bloch, "Über die Quantenmechanik der Elektronen in Kristallgittern," Z. Phys. 52, 555 (1928); C. Zener, "A theory of the electrical breakdown of solid dielectrics", Proc. R. Soc. A 145, 523 (1934).
[2] J. Feldmann, K. Leo, J. Shah, D. B. A. Miller, J. E. Cunningham, S. Schmitt-Rink, T. Meier, G. von Plessen, A. Schulze, and P. Thomas, "Optical investigation of Bloch oscillations in a semiconductor superlattice," Phys. Rev. 

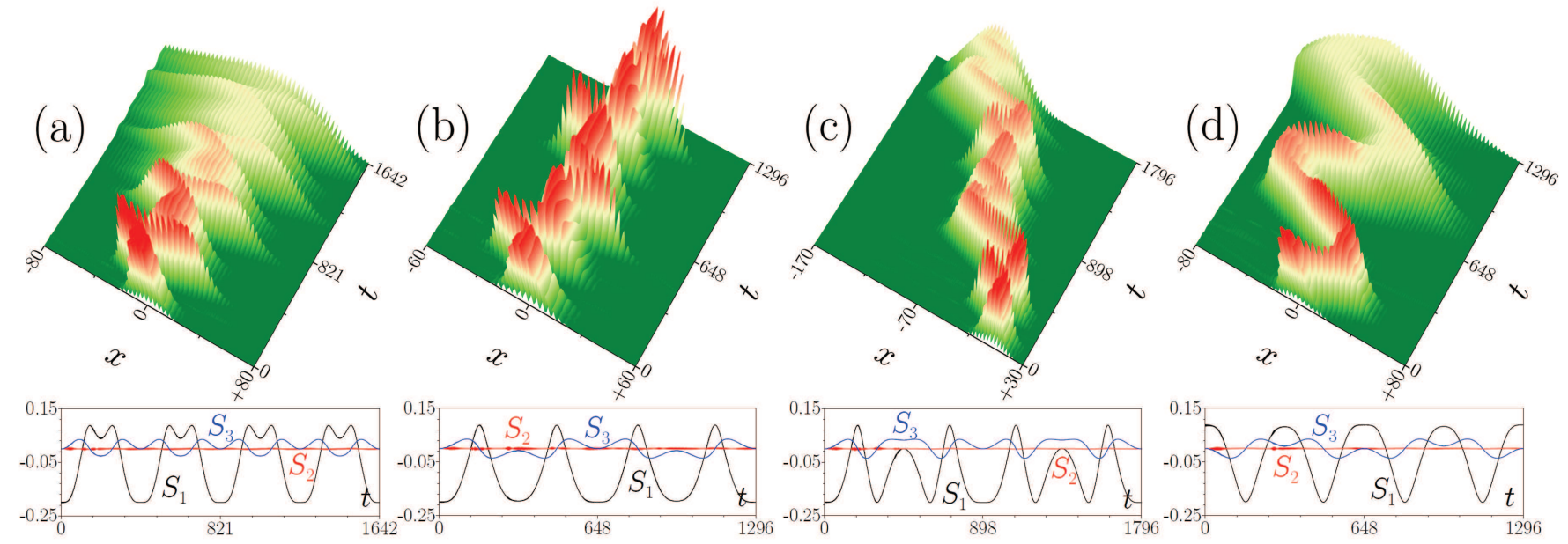

FIG. 8. (Color online) Evolution dynamics for the first-band excitations at (a) $\omega=0.0153$, (b) $\omega=0.0097$, (c) $\omega=0.0070$, and of the second-band excitation at $(\mathrm{d}) \omega=0.0097$ in the ZL at $\gamma=2$. Only $\left|\psi_{1}\right|$ component is shown. Second row shows corresponding expectation values of the spin components. In all cases $d(t=0)=7 \pi$. In panels (b)-(d) evolution time corresponds to two complete oscillation periods, while in (a) it corresponds to eight oscillation periods.

B 467252 (1992); K. Leo, H. Bolivar, F. Brüggemann, R. Schwedler, and K. Köhler, "Observation of Bloch oscillations in a semiconductor superlattice," Solid State Commun. 84, 943 (1992); C. Waschke, H. G. Roskos, R. Schwedler, K. Leo, H. Kurz, and Köhler, "Coherent submillimeter-wave emission from Bloch oscillations in a semiconductor superlattice," Phys. Rev. Lett. 70, 3319 (1993).

[3] O. Morsch, J. H. Müller, M. Cristiani, D. Ciampini, and E. Arimondo, "Bloch oscillations and mean-field effects of Bose-Einstein condensates in 1D optical lattices," Phys. Rev. Lett. 87, 140402 (2001); M. Cristiani O. Morsch, J. H. Müller, D. Ciampini, and E. Arimondo, "Experimental properties of Bose-Einstein condensates in onedimensional optical lattices: Bloch oscillations, LandauZener tunneling, and mean-field effects," Phys. Rev. A 65, 063612 (2002).

[4] F. K. Abdullaev, B. B. Baizakov, S. Darmanyan, V.V. Konotop, and M. Salerno, "Nonlinear excitations in arrays of Bose-Einstein condensates," Phys. Rev. A 64, 043606 (2001).

[5] U. Peschel, T. Pertsch, and F. Lederer, "Optical Bloch oscillations in waveguide arrays," Opt. Lett. 23, 1701 (1998); T. Pertsch, P. Dannberg, W. Elflein, A. Bräuer, and F. Lederer, "Optical Bloch oscillations in temperature tuned waveguide arrays," Phys. Rev. Lett. 83, 4752 (1999); R. Morandotti, U. Peschel, J. S. Aitchison, H. S. Eisenberg, and Y. Silberberg, "Experimental observation of linear and nonlinear optical Bloch oscillations," Phys. Rev. Lett. 83, 4756 (1999); R. Sapienza, P. Costantino, D. Wiersma, M. Ghulinyan, C. J. Oton, and L. Pavesi, "Optical analogue of electronic Bloch oscillations," Phys. Rev. Lett. 91, 263902 (2003); H. Trompeter, W. Krolikowski, D. N. Neshev, A. S. Desyatnikov, A. A. Sukhorukov, Y. S. Kivshar, T. Pertsch, U. Peschel, and F. Lederer, "Bloch oscillations and Zener tunneling in two-dimensional photonic lattices," Phys. Rev. Lett. 96, 053903 (2006).
[6] G. Nenciu, "Dynamics of band electrons in electric and magnetic fields: rigorous justification of the effective Hamiltonians," Rev. Mod. Phys. 63, 91 (1991); M. Glück, A. R. Kolovsky, and H. J. Korsch, "Wannier-Stark resonances in optical and semiconductor superlattices," Phys. Rep. 366, 103 (2002); A. Kolovsky and H. Korsch, "Bloch oscillations of cold atoms in optical lattices," Int. J. Mod. Phys. B 18, 1235 (2004).

[7] D. H. Dunlap and V. M. Kenkre, "Dynamic localization of a charged particle moving under the influence of an electric field," Phys. Rev. B 34, 3625 (1986).

[8] M. Holthaus, "Collapse of minibands in far-infrared superlattices," Phys. Rev. Lett. 69, 351 (1992).

[9] A. Eckardt, C. Weiss, and M. Holthaus, "Superfluidinsulator transition in a periodically driven optical lattice," Phys. Rev. Lett., 95, 260404 (2005); A. Zenesini, H. Lignier, D. Ciampini, O. Morsch, and E. Arimondo, "Coherent control of dressed matter waves," Phys. Rev. Lett. 102, 100403 (2009).

[10] S. Longhi, M. Marangoni, M. Lobino, R. Ramponi, P. Laporta, E. Cianci, and V. Foglietti, "Observation of dynamic localization in periodically curved waveguide arrays," Phys. Rev. Lett. 96, 243901 (2006); R. Iyer, J. S. Aitchison, J. Wan, M. M. Dignam, and C. M. de Sterke, "Exact dynamic localization in curved AlGaAs optical waveguide arrays," Opt. Express 15, 3212 (2007); F. Dreisow, M. Heinrich, A. Szameit, S. Döring, S. Nolte, A. Tünnermann, S. Fahr, and F. Lederer, "Spectral resolved dynamic localization in curved fs laser written waveguide arrays," Opt. Express 16, 3474 (2008).

[11] F. Dreisow, Y. V. Kartashov, M. Heinrich, V. A. Vysloukh, A. Tünnermann, S. Nolte, L. Torner, S. Longhi, and A. Szameit, "Spatial light rectification in an optical waveguide lattice," Europhys. Lett. 101, 44002 (2013); Y. V. Kartashov, V. A. Vysloukh, V. V. Konotop, and L. Torner, "Diffraction control in PT-symmetric photonic lattices: from beam rectification to dynamic localization," Phys. Rev. A 93, 013841 (2016). 
[12] M. Ablowitz and J. Ladik, "Nonlinear differentialdifference equations," J. Math. Phys. 16, 598 (1975).

[13] M. Bruschi, D. Levi, and O. Ragnisco, "Discrete version of the nonlinear Schrödinger equation with linearly $x$ dependent coefficients," Nuovo Cimento A 53, 21 (1979).

[14] V. V. Konotop, O. A. Chubykalo, and L. Vázquez, "Dynamics and interaction of solitons on an integrable inhomogeneous lattice," Phys. Rev. E 48, 563 (1993).

[15] Yu. V. Bludov, V. V. Konotop, and M. Salerno, "Dynamical localization of gap-solitons by time periodic forces," EPL 87, 20004 (2009); Y. V. Bludov, V. V., Konotop, and M. Salerno, "Long-lived matter wave Bloch oscillations and dynamical localization by time-dependent nonlinearity management," J. Phys. B: At. Mol. Opt. Phys. 42, 105302 (2009).

[16] H. Lignier, C. Sias, D. Ciampini, Y. Singh, A. Zenesini, O. Morsch, ane E. Arimondo, "Dynamical Control of Matter-Wave Tunneling in Periodic Potentials," Phys. Rev. Lett. 99, 220403 (2007); A. Eckardt, M. Holthaus, H. Lignier, A. Zenesini, D. Ciampini, O. Morsch, and E. Arimondo, "Exploring dynamic localization with a BoseEinstein condensate," Phys. Rev. A 79, 013611 (2009).

[17] Y-J. Lin, R. L. Compton, K. Jiménez-García, W. D. Phillips, J. V. Porto, and I. B. Spielman, "A synthetic electric force acting on neutral atoms," Nature Phys. 7, 531 (2011).

[18] Y.-J. Lin, R. L. Compton, K. Jiménez-García, J. V. Porto, and I. B. Spielman, "Synthetic magnetic fields for ultracold neutral atoms," Nature 462, 628 (2009).

[19] M. Atala, M. Aidelsburger, J. T. Barreiro, D. Abanin, T. Kitagawa, E. Demler, and I. Bloch, "Direct measurement of the Zak phase in topological Bloch bands," Nat. Phys. 9, 795 (2013); C. J. Kennedy, G. A. Siviloglou, H. Miyake, W. C. Burton, and W. Ketterle, "Spin-orbit coupling and quantum spin Hall effect for neutral atoms without spin flips," Phys. Rev. Lett. 111, 225301 (2013).
[20] J. Dalibard, F. Gerbier, G. Juzeliūnas, and P. Öhberg, "Artificial gauge potentials for neutral atoms," Rev. Mod. Phys. 83, 1523 (2011).

[21] Y. J. Lin, K. Jiménez-García, and I. B. Spielman, "Spinorbit-coupled Bose-Einstein condensates," Nature 471, 83 (2011).

[22] V. Galitski and I. B. Spielman, "Spin-orbit coupling in quantum gases," Nature 494, 49 (2013); X. Zhou, Y. Li, Z. Cai, and C. Wu, "Unconventional states of bosons with synthetic spin-orbit coupling," J. Phys. B: At. Mol. Opt. Phys. 46, 134001 (2013).

[23] K. Jiménez-García, L. J. LeBlanc, R. A. Williams, M. C. Beeler, A. R. Perry, and I. B. Spielman, "Peierls substitution in an engineered lattice potential," Phys. Rev. Lett. 108, 225303 (2012).

[24] D. Witthaut, "Quantum walks and quantum simulations with Bloch-oscillating spinor atoms," Phys. Rev. A 82, 033602 (2010).

[25] J. Larson, J.-P. Martikainen, A. Collin, and E. Sjöqvist, "Spin-orbit-coupled Bose-Einstein condensate in a tilted optical lattice," Phys. Rev. A 82, 043620 (2010).

[26] Y. Ke, X. Qin, H. Zhong, J. Huang, C. He, and C. Lee, "Bloch-Landau-Zener dynamics in single-particle Wannier-Zeeman systems," Phys. Rev A 91, 053409 (2015).

[27] Y. V. Kartashov, V. V. Konotop, D. A. Zezyulin, and L. Torner, "Bloch oscillations in optical and Zeeman lattices in the presence of spin-orbit coupling", arXiv:1610.04716. to appear in Phys. Rev. Lett.

[28] W. V. Houston, "Acceleration of electrons in a crystal lattice," Phys. Rev. 57, 184 (1940).

[29] Y. Zhang and C. Zhang, "Bose-Einstein condensates in spin-orbit-coupled optical lattices: Flat bands and superfluidity," Phys. Rev. A 87, 023611 (2013).

[30] M. Born and V. A. Fock, "Beweis des Adiabatensatzes," Zeitschrift für Physik A 51, 165 (1928). 